\title{
A study of ageing in timing RPCs
}

\author{
L. Lopes ${ }^{\mathrm{a}, \mathrm{b}}$, R. Ferreira-Marques ${ }^{\mathrm{a}, \mathrm{b}}$, P. Fonte ${ }^{\mathrm{a}, \mathrm{c}, *}$, A.P. Piedade ${ }^{\mathrm{d}}$, A. Policarpo ${ }^{\mathrm{a}, \mathrm{b}}$ \\ ${ }^{a}$ LIP_Laboratório de Instrumentação e Física Experimental de Particulas, 3004-516 Coimbra, Portugal \\ ${ }^{\mathrm{b}}$ CFRM, Departamento de Física da Universidade de Coimbra, 3004-516 Coimbra, Portugal \\ ${ }^{\mathrm{c}}$ Instituto Superior de Engenharia de Coimbra, 3030-199 Coimbra, Portugal \\ ${ }^{\mathrm{d}}$ ICEMS, Departamento de Engenharia Mecânica, Universidade de Coimbra, 3030-201 Coimbra, Portugal
}

Available online 21 July 2004

\begin{abstract}
The present study concerns the search for ageing effects in $0.3 \mathrm{~mm}$ gap timing RPCs. Counters were made with glass and aluminium electrodes and kept under a continuous gas flow of tetrafluorethane with $10 \%$ sulphur hexafluoride, $5 \%$ isobutane and water at $10 \%$ relative humidity. After 500 days of operation at the saturation current an accumulated charge of $800 \mathrm{mC}$ was collected, equivalent to over 8 years of continuous operation at $300 \mathrm{~Hz} / \mathrm{cm}^{2}$. No evidence was found of any long-term increase of dark current, suggesting that the ageing effect in timing RPCs is less severe than in streamer-mode glass RPCs. However, a visual inspection of the electrodes confirmed the existence of a deposit over the glass electrodes that was analysed by Electron Probe Microanalysis.
\end{abstract}

(C) 2004 Elsevier B.V. All rights reserved.

PACS: $29.40 \mathrm{Cs}$

Keywords: RPC; TOF; Ageing

\section{Introduction}

Severe ageing of glass RPCs operating in streamer mode has been reported [1,2] and related to the presence of water vapour traces in the gas mixture. An unidentified deposit was found over the glass surface, severely increasing

\footnotetext{
*Corresponding author. LIP-Combra, Departamento de fisica da Universidade de Coimbra, 3004-516 Coimbra, Portugal. Tel: +351-239-833-465; fax: +351-23-98-29-158.

E-mail address: fonte@lipc.fis.uc.pt (P. Fonte).
}

the dark count rates and reducing the counter efficiency.

Naturally, the investigation of such effect in timing RPCs, often made with glass electrodes and working in somewhat similar gaseous mixtures is of considerable practical importance.

\section{Experimental setup}

The test setup comprised six $9 \mathrm{~cm}^{2}$ single-gap counters, each made with one glass (SCHOTT 
ATHERMAL ${ }^{\circledR}$ tainted glass) and one aluminium electrode. Three counters had a glass cathode and three had an aluminium cathode.

All counters were illuminated laterally, along the gas gap, by a DC mercury UV lamp that liberated primary electrons by photoelectric effect at the cathodes. The effectively illuminated region had an area of approximately $1 \mathrm{~cm}^{2}$, recognized by the deposits found over the glass electrode (see below, Fig. 1).

The different (glass or aluminium) cathode photosensitivities were compensated by slightly adjusting the applied voltages, ranging from 2.8 to $3.0 \mathrm{kV}$, until all chambers shown similar current values, around $20 \mathrm{nA}$. For the settings chosen the currents were essentially independent from any small variations in the lamp intensity, having reached the maximum value allowed by the resistive electrode.

The counters were illuminated for $22 \mathrm{~h}$ and kept in darkness for $2 \mathrm{~h}$ per day, counter currents, internal temperature, atmospheric pressure and lamp intensity being continuously recorded.

The setup was kept in a continuous flow of "standard" timing RPC gas, $\mathrm{C}_{2} \mathrm{H}_{2} \mathrm{~F}_{4}+10 \% \mathrm{SF}_{6}+$ $5 \%$ iso- $\mathrm{C}_{4} \mathrm{H}_{10}$, also enough water vapour being added to keep a $10 \%$ relative humidity. The gas container was made of acrylic plastic with Viton O-ring seals. Several common types of glues, plastics and metals were used internally as needed.
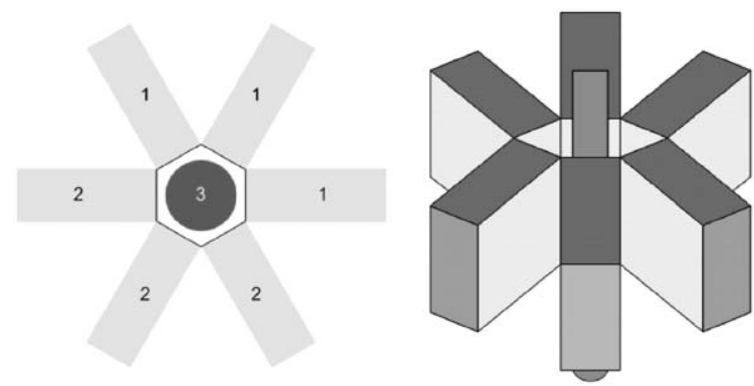

Fig. 1. Schematic drawing of the experimental setup. The chambers where illuminated laterally, along the gas gap, by a mercury UV lamp (1 - chambers with aluminium cathode; 2chambers with glass cathode; 3-DC mercury UV lamp).

\section{Dark current}

As an increase in dark current was the main experimental indication of ageing reported in Ref. [1], we use this parameter as indicative of an eventual ageing effect.

Fig. 2 shows the dark current for each of the six chambers averaged over the second of the two daily rest hours. There is no evidence of any systematic increase of dark current with time, suggesting that the ageing effect mentioned above is less severe in timing RPCs than in streamer-mode RPCs. The short-term dark current fluctuations are well correlated for the three chambers of the same type and are of environmental origin (mostly temperature).

When the cathode is made of aluminium, the new chambers apparently need to work for a longer time until the dark current stabilises. A possible explanation may be related to the different surface porosity of aluminium and glass, the former easily trapping microscopic dust particles that generate dark currents. Nevertheless, there is a general tendency for the initial dark currents to decrease with time.

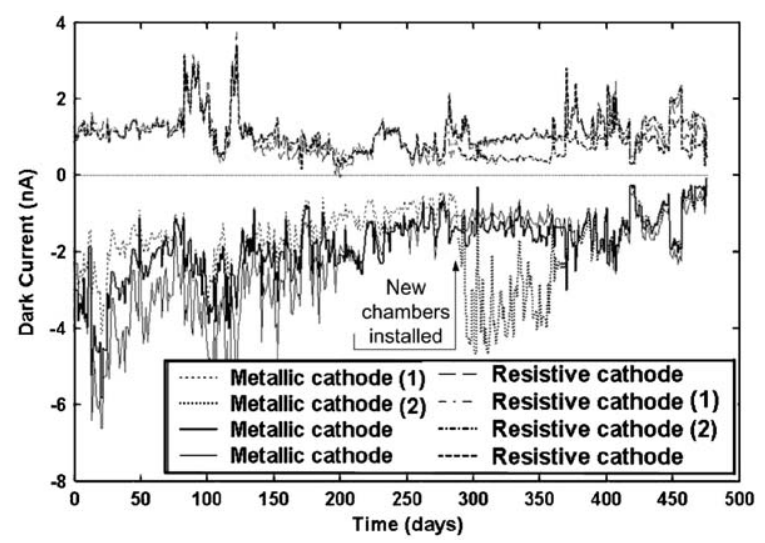

Fig. 2. Dark current as a function of operation time. No systematic increase in dark current is apparent (positive current: glass cathode; negative current: glass anode). After 280 days two counters (1), one of each configuration, were removed for surface analysis, and replaced by two new chambers (2). 


\section{Integrated charge}

Fig. 3 shows the charge accumulated in each chamber after 480 days of operation. Two chambers were removed for surface analysis at day 280. The replacement chambers had the tainted glass substituted by an ordinary sheet glass.

Considering an average avalanche charge of $10 \mathrm{pC}$, the accumulated charge of $800 \mathrm{mC}$ corresponds to about $80 \times 10^{9}$ avalanches in each chamber. As the effectively illuminated area in each chamber is about $1 \mathrm{~cm}^{2}$, one estimates that the chambers under test operate at a counting rate of about $2.5 \mathrm{kHz} / \mathrm{cm}^{2}$.

The accumulated charge corresponds to 8.5 years of operation at a typical maximum counting rate of $300 \mathrm{~Hz} / \mathrm{cm}^{2}$.

\section{Surface composition analysis by electron probe microanalysis (EPMA)}

After 280 days of operation two chambers were opened for analysis. A visual inspection of the electrodes revealed a multicoloured dry deposit over the glass electrodes. The colours appeared to be merely due to light interference and not intrinsic to the material.

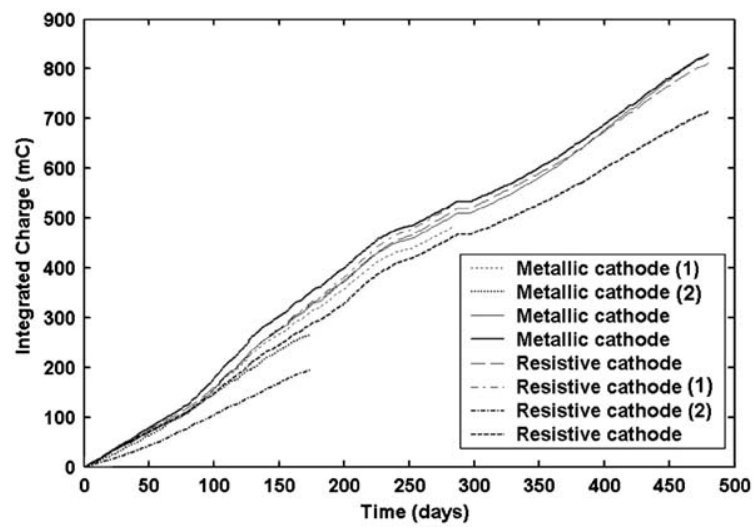

Fig. 3. Integrated charge as a function of operation time. Two chambers (1) were substituted after 280 days by new ones (2). The remaining four chambers accumulated an integrated charge of about $800 \mathrm{mC}$.
When the glass was operated as a cathode, the deposit was well localized over an area of approximately $1 \mathrm{~cm}^{2}$ close to the UV-light entrance slit, presumably corresponding to the effectively illuminated region. When the glass was operated as an anode the deposit was distributed over most of the electrode area.

The aluminium electrode facing the glass cathode appeared covered with a localised matte layer that coincided with the deposit on the glass. The aluminium electrode facing the glass anode seemed completely clean.

In Table 1 we present a qualitative EPMA of the most abundant elements found in the surfaces of the glass electrodes (anode and cathode) and, for comparison, on both surfaces of a new glass. Only the relative concentrations within the same element are meaningful and no comparisons should be made along the table columns.

The surfaces in contact with the gas show a significant enrichment in fluor, oxygen and sulphur contents after irradiation, presumably by deposition from the gas (oxygen may be also extracted from the glass). These elements may eventually be associated with lighter ones, but the method could not detect elements lighter than oxygen.

There is also some surface enrichment in elements that are not present in the gas. For certain elements the enrichment is observed both on glass anodes and cathodes $(\mathrm{Si}, \mathrm{Al}, \mathrm{Ca}, \mathrm{Mg})$ or only on one electrode polarity $(\mathrm{Cl}, \mathrm{Na}, \mathrm{Fe})$. Naturally aluminium could have been etched from the opposing electrode and transported by the avalanches across the gap. The enrichment observed for the remaining elements could be a consequence of the ion flow in the glass due to the electric current. However, this process does not explain why the enrichment appears both in the anode and cathode surfaces or why there is no corresponding depletion on the back surfaces.

In Table 1 is also shown a similar analysis of the aluminium electrodes. There is also an enrichment in fluor for both electrode polarities. However, the aluminium cathode seems to have been somewhat cleaned of initial traces of oxygen and silicon, while the anode appeared enriched not only in oxygen but also in potassium and sodium. 
Table 1

Qualitative EPMA of the most abundant elements found in the surfaces of two glass electrodes and, for comparison, in both surfaces of a new, never used, glass

\begin{tabular}{|c|c|c|c|c|c|c|}
\hline \multicolumn{7}{|l|}{ GLASS } \\
\hline Element & New glass face 1 & New glass face 2 & Cathode back face & Cathode & Anode back face & Anode \\
\hline $\mathrm{F}$ & 0 & 0 & 81 & 669 & 0 & 228 \\
\hline $\mathrm{O}$ & 438 & 360 & 505 & 1002 & 627 & 890 \\
\hline $\mathrm{Cl}$ & 0 & 0 & 0 & 0 & 0 & 84 \\
\hline $\mathrm{S}$ & 0 & 0 & 0 & 7 & 0 & 5 \\
\hline $\mathrm{Si}$ & 29 & 53 & 84 & 536 & 170 & 1052 \\
\hline $\mathrm{Al}$ & 4 & 7 & 7 & 55 & 11 & 44 \\
\hline $\mathrm{Mg}$ & 4 & 5 & 7 & 29 & 11 & 17 \\
\hline $\mathrm{Na}$ & 8 & 13 & 44 & 55 & 11 & 344 \\
\hline $\mathrm{Fe}$ & 84 & 60 & 78 & 184 & 96 & 91 \\
\hline $\mathrm{Ca}$ & 0 & 0 & 0 & 40 & 0 & 66 \\
\hline \multicolumn{7}{|c|}{ ALUMINIUM } \\
\hline Element & New & Cathode & Anode & & & \\
\hline $\mathrm{F}$ & 0 & 125 & 302 & & & \\
\hline $\mathrm{O}$ & 99 & 50 & 133 & & & \\
\hline $\mathrm{Si}$ & 27 & 9 & 26 & & & \\
\hline $\mathrm{K}$ & 0 & 0 & 12 & & & \\
\hline $\mathrm{Na}$ & 0 & 0 & 14 & & & \\
\hline $\mathrm{Al}$ & 5827 & 6094 & 5274 & & & \\
\hline
\end{tabular}

Elements lighter than oxygen were not detected and the relative concentrations between different elements are arbitrary. Similar analyses were also performed on the aluminium electrodes.

Clearly a full understanding of the ion flows in aluminium-glass RPCs is not available at this moment.

Fig. 4 shows the X-ray elemental distribution, obtained through EPMA, of the fluor concentration over a small region of the glass (cathode) surface.

\section{Conclusions}

Ageing studies were performed on six $0.3 \mathrm{~mm}$ gap timing RPCs made with glass and aluminium electrodes and operating in a mixture of tetrafluorethane with $10 \%$ sulphur hexafluoride, $5 \%$ isobutane and water at $10 \%$ relative humidity.

After a charge transfer of about $800 \mathrm{mC}$, equivalent to 8.5 years of normal operation, no systematic increase of dark current was detected, suggesting that no severe ageing process is at work.

However, some thin deposits were found over the electrodes. An EPMA qualitative analysis

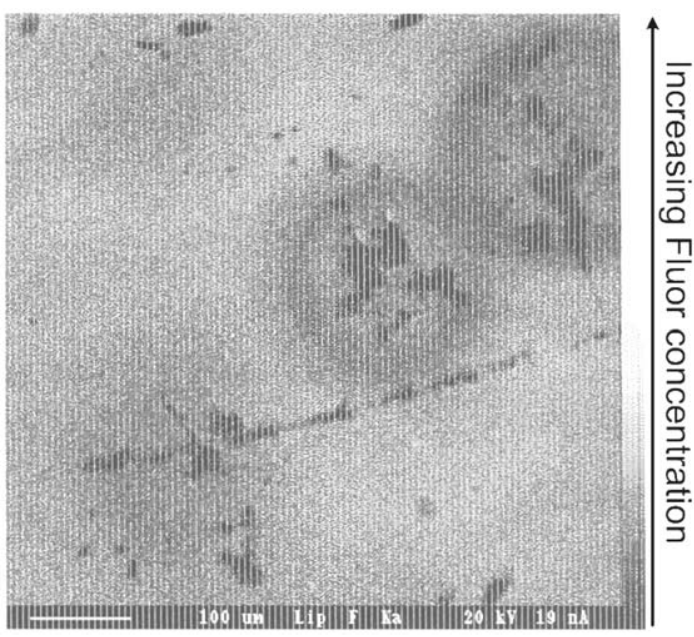

Fig. 4. X-ray elemental distribution, obtained through EPMA, of the fluor concentration over the surface of a glass cathode.

suggested that the glass surfaces contained mainly fluor and oxygen, with enrichment in several trace metals and in chlorine. However, a full under- 
standing of all the perceived ion flows is not available at this moment.

\section{Acknowledgements}

We were warmly welcomed to ICEMS, Departamento de Engenharia Mecânica da Universidade de Coimbra, by its coordinator Prof. Maria Teresa Freire Vieira.

Alberto Blanco Castro contributed many useful suggestions and Nuno Carolino and Américo
Pereira provided the usual competent technical support.

This work was financed by Fundação para a Ciência e Tecnologia project POCTI/FNU/49513/ 2002.

\section{References}

[1] H. Sakai, et al., Nucl. Instr. and Meth. A 484 (2002) 153.

[2] A. Candela, et al., Ageing and recovering of glass RPC, Nucl. Instr. and Meth. A, (2004) these proceedings. 